population has OA. This study aimed to analyze the indications and average doses prescribed in the treatment of knee osteoarthritis in Primary Care in Spain.

Objectives: This study aimed to analyze the comorbidity profile of men and women affected by symptomatic knee OA and the differences between genders in the perception of health status with regard to pain and loss of function due to their OA, quality of life and mental health.

Methods: The EMARTRO study was designed as an observational, multicenter, transversal study to compare probability of suffering a comorbidities based on presence of symptomatic knee OA visited by GPs. Sociodemographic, anthropometric, clinical parameters and clinical variables of interest were recorded. The probability of suffering comorbidities based on presence OA was estimated using the Odds Ratio estimation with conditioned logistic regression models. Depending on the variable, comparisons between groups were done using t-Student, Chi-square and Mann-Whitney.

Results: A total of 646 patients were included, $71 \%$ were women. Mean (SD) age was $67.9(6.6)$ years.

Patients were obese without gender differences, with a BMI of 30.4 (4.4) and 31.2 (5.5) $(p=0.0651)$ in men and women, respectively.

Men had a mean (SD) systolic blood pressure higher than women, 134.7 (15.0) vs $132.2(14.5) \mathrm{mmHg}(\mathrm{p}=0.0453)$ and no differences were observed in diastolic pressure $(p=0.5930)$

As regards to the concomitant pathologies, no increase was detected in the likelihood of suffering comorbidities linked to the gender [OR $=0.607(95 \% \mathrm{Cl}$ : $0.260-1.418) p=0.2490]$. Men with $O A$ were more likely to have angina pectoris [OR=4.493 (95\% Cl: 1.299-15.536) $\mathrm{p}=0.0176]$ and underwent coronary bypass [OR=3.706 (95\% Cl: 1.389-9.890) p=0089]

Osteoarthritic women elicited more pain in Huskisson's VAS 62.8 (14.7) vs 66.8 (15.4) $\mathrm{mm}(\mathrm{p}=0.0027)$ and worse function according to the Lequesne index 10.0 (4.9) vs $12.1(4.7)(p<0.0001)$

In terms of quality of life according to the EuroQol, women presented worse quality of life in dimesion of mobility $(p=0.0001)$ and in the dimensions of daily activities, pain/discomfort and anxiety/depression $(p<0.0001)$.

Finally, regarding mental health status, women had worse scores in the Goldberg scale to detect psychological disorders $(p<0.0001)$ and more cases of anxiety and depression according to the HAD scale $(p<0.0001)$.

Conclusions: The results of the present study indicate that women with osteoarthritis of the knee, despite having the same diagnosis and a similar comorbidity profile to men, have a worse perception of health status regarding the symptoms of their osteoarthritis, their quality of life and their mental health.

Disclosure of Interest: M. Herrero Barbero Employee of: Bioiberica, S. Gimenez: None declared, J. Vergara: None declared, E. Viles I Lladó Employee of: Bioiberica, H. Martinez Employee of: Bioiberica, G. Rodríguez Roca: None declared, L. Sánchez Employee of: Bioiberica, J. A. Díaz Muñoz: None declared, J. F. Frias: None declared, A. Castaño: None declared, J. J. Jiménez Díaz: None declared, Á. Rodríguez de Cossío: None declared, R. Belenguer: None declared, J. Vergés Milano Consultant for: Bioiberica, Employee of: Bioiberica, J. L. Llisterri: None declared

DOI: 10.1136/annrheumdis-2017-eular.3625

\section{AB1148 INFODEMIOLOGY AND SEASONALITY OF SYSTEMIC LUPUS ERYTHEMATOUS USING GOOGLE TRENDS}

M. Radin, S. Sciascia. Department of Clinical and Biological Sciences, Center of Research of Immunopathology and Rare Diseases- Coordinating Center of Piemonte and Valle d'Aosta Network for Rare Diseases, Torino, Italy

Background: People affected by chronic rheumatic conditions, such as systemic lupus erythematosus (SLE), frequently rely on Internet and search engines to look for terms related to their disease, and its possible causes, symptoms and treatments. "Infodemiology" and "infoveillance" are two recent terms created to describe a new developing approach for public health, based on Big Data monitoring and data mining ${ }^{1}$. Different clinical and epidemiological studies have been conducted on seasonality of SLE, and focused on flare, fatigue and periodicity of onset of clinical manifestations. Overall, SLE disease activity has been associated with specific seasonal patterns in both, Northern and Southern hemispheres, possibly in relation to sun exposure, meteorological factors and vitamin D levels ${ }^{2,3}$.

Objectives: In this study, we aimed to investigate trends of Internet research linked to SLE and symptoms associated to the disease seasonality by applying a Big Data monitoring approach.

Methods: We analyzed the large amount of data generated by Google Trends, considering "lupus", "relapse" and "fatigue" in a 10-year web-based research. Google Trends automatically normalized data for the overall number of searches, and presented them as relative search volumes, in order to compare variations of different search terms across regions and periods. The Menn-Kendall test was used to evaluate the overall seasonal trend of each search term and possible correlation between search terms.

Results: We observed a seasonality for Google search volumes for lupus-related terms (Figure 1). In Northern hemisphere, relative search volumes for "lupus" were correlated with "relapse" $(\tau=0.85 ; p=0.019)$ and with fatigue $(\tau=0.82 ; p=0.003)$, whereas in Southern hemisphere we observed a significant correlation between "fatigue" and "relapse" ( $\tau=0.85 ; p=0.018)$. Similarly, a significant correlation
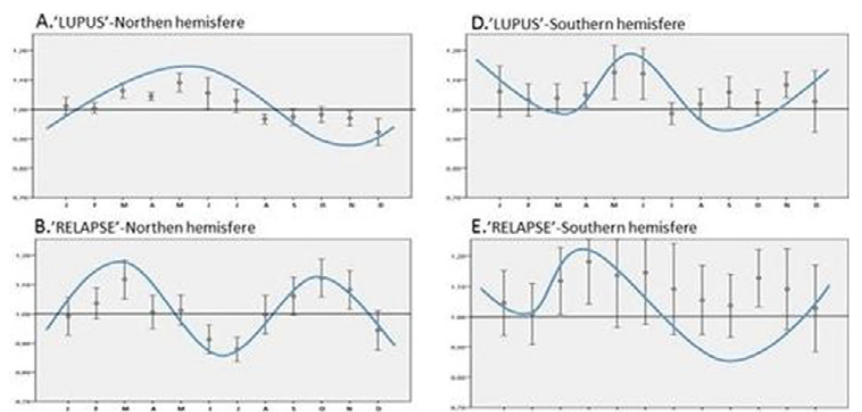

C.'FATIGUE' Northen hemisfere

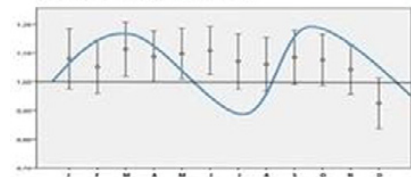

F.'FATIGUE'-Southern hemisfere

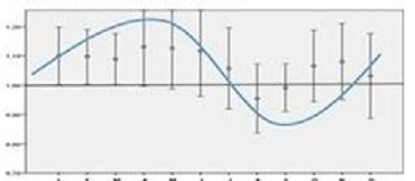

Profile plots for relative search volumes. Profile plots show the relative search volumes for 'lupus' ( $A$ and $D)$, 'relapse' ( $B$ and $E$ ) and 'fatigue' ( $C$ and $F$ ), in the Northern and Southern hemispheres, respectively, for different months of the year, with estimates sinusoidal curves.

between "fatigue" and "relapse" ( $\tau=0.70 ; p<0.001)$ was seen also in the Northern hemisphere.

Conclusions: Despite the intrinsic limitations of this approach, the current study provides additional evidence for seasonality of lupus by using Google Trends. Internet-acquired data might represent a real-time surveillance tool and an alert for healthcare systems in order to plan the most appropriate resources in specific moments with higher disease burden

References:

[1] Eysenbach G. Infodemiology and infoveillance: framework for an emerging set of public health informatics methods to analyze search, communication and publication behavior on the Internet. J Med Internet Res. 2009;11:e11.

[2] Dall'Ara F, Andreoli L, Piva N, Piantoni S, Franceschini F, Tincani A. Winter lupus flares are associated with low vitamin D levels in a retrospective longitudinal study of Italian adult patients. Clin Exp Rheumatol. 33:153-8.

[3] Zhang H, Xu S, Tang D, Liang D, Liu H. Seasonal distribution of active systemic lupus erythematosus and its correlation with meteorological factors. 2011:66:1009-1013.

Acknowledgements: None.

Disclosure of Interest: None declared

DOI: 10.1136/annrheumdis-2017-eular.1603

\section{AB1149 THE ABILITY OF THE HEALTH SYSTEM TO IDENTIFY THE BURDEN OF RHEUMATOID ARTHRITIS IN SERBIA: A EULAR SURVEY}

M.I. Zlatkovic-Svenda ${ }^{1}$, R.M. Stojanovic ${ }^{1}$, S.B. Sipetic-Grujicic ${ }^{2}$, M.M. Radak-Perovic ${ }^{1}$, N.S. Damjanov ${ }^{1}$, F. Guillemin ${ }^{3} .{ }^{1}$ Institute of Rheumatology University of Belgrade School of Medicine; ${ }^{2}$ Institute of Epidemiology University of Belgrade School of Medicine, Belgrade, Serbia; ${ }^{3}$ INSERM - Cic-Ec 1433, University Hospital, and Université de Lorraine, Ea 4360 Apemac, Nancy, France

Objectives: to estimate the rheumatoid arthritis (RA) prevalence in two urban regions of Serbia, covering the northern and the southern part, under the European League Against Rheumatism (EULAR) prevalence survey; to assess the ability of the health system to recognize and treat patients with RA.

Methods: The survey was conducted in four Serbian towns: Belgrade in the north and three towns in the south: Cacak (Moravicki region), Uzice (Zlatiborski region) and Krusevac (Rasinski region), covering $36.5 \%$ of the total Serbian population with more than $99 \%$ Caucasians, mostly orthodox Serbs (83\%), <4\% Hungarians, Roms and Bosnians and a minority of other nationalities. The first-detection phase of the study comprised previously translated and validated telephone Questionnaire usage with 33 items covering signs, symptoms, selfreported diagnosis and classification criteria for RA (ACR 1987) (1). Diagnoses were confirmed by rheumatologists in a second-confirmation phase. Prevalence results were standardized for age and sex with regard to Serbian population (national census 2002). Confirmed RA cases were asked two more questions: "How long had you had symptoms before you were given the diagnosis of RA" and "How had you been treated for that period of time".

Results: 6213 people were contacted and $63.6 \%$ answered the survey; joint pain was reported by 1,799 persons, and joint pain accompanied with joint swelling by 606 persons. A total of 23 RA cases were identified; 2 newly diagnosed. The standardized RA prevalence estimates were $0.30 \%$ (95\% confidence interval $[95 \% \mathrm{Cl}] 0.09 ; 0.51)$ for the north, e.g. $0.09(95 \% \mathrm{Cl} 0.08 ; 0.26)$ for men and $0.49 \%$ $(95 \% \mathrm{Cl} 0.19 ; 0.79)$ for women. RA prevalence estimates were $0.42 \%(0.12 ; 0.72)$ for the south; $0.28(0.00 ; 0.56)$ for men and $0.55 \%(0.09 ; 1.00)$ for women, with 
the female to male ratio 5,5:1 in the north and 2:1 in the south. Time period from the first symptoms occurence to the RA diagnosis was 17.7 (13.2) months for the northern part and 25.0 (16.9) for the southern; 20.6 (14.9) for Serbia; in that period patients were mostly treated with NSAIDS (82\%) and physical therapy $(30 \%)$; short-lasting corticosteroids were given to $13 \%$, peroral corticosteroids to $4 \%$ and no patients were treated with DMARDS.

Conclusions: RA prevalence in the southern and northern part of Serbia is in line $(0.42 \%$ [95\% $\mathrm{Cl} 0.12 ; 0.72])$ vs $0.30 \%$ [ $95 \% \mathrm{Cl} 0.09 ; 0.51]$ ), being more frequently presented in females as compared to males (five times more in the north and two times more in the south). Delay in diagnosis as compared to the first symptoms occurence was 21 months and during that time no patients were treated with DMARDS.

References:

[1] Zlatkovic-Svenda MI, Stojanovic RM, Milenkovic MP, Vlajinac HD, Le Bihan E, Guillemin F. Adaptation and validation of a telephone questionnaire - Serbian version for case detection of rheumatoid arthritis and spondyloarthropathy (multicentric Eular study). Clin Exp Rheumatol. 2007;25 (1): 75-84.

Disclosure of Interest: None declared

DOI: 10.1136/annrheumdis-2017-eular.4899

\section{AB1150 SLEEP HEALTH AND QUALITY OF LIFE IN PATIENTS WITH KNEE OSTEOARTHRITIS BEFORE AND AFTER TOTAL KNEE REPLACEMENT}

M. Nuñez ${ }^{1}$, E. Nuñez ${ }^{2}$, J.M. Segur ${ }^{3}$, L. Lozano ${ }^{3}$, J. Montañana ${ }^{3}$, V. Segura ${ }^{3}$ M. Marti ${ }^{4}$, A. Garcia-Cardó ${ }^{4}$, S. Sastre ${ }^{3}$, X. Alemany ${ }^{3} .{ }^{1}$ Rheumatology and IDIBAPS Area 1, Hospital Clinic; ${ }^{2}$ SAP Suport al Diagnòstic i al Tractament, Institut Català de la Salut; ${ }^{3}$ Orthopedic Surgery and IDIBAPS; ${ }^{4}$ Rheumatology, Hospital Clinic, Barcelona, Spain

Background: Studies report that sleep disturbances are often associated with chronic musculoskeletal disease. There is no agreed definition of sleep health, but some characteristics, such as sleep duration (number of hours daily) and sleep quality or satisfaction (subjective evaluation of good or poor sleep) are used to evaluate sleep health. In a previous study in patients with severe osteoarthritis awaiting total knee replacement (TKR), patients reporting good quality sleep had better health-related quality of life (HRQL) measured by the specific WOMAC and generic SF-36 questionnaires.

Objectives: To measure sleep health in patients included on a waiting list for TKR and 12 months after TKR.

Methods: Prospective study with a 12-month follow up. Sociodemographic and clinical variables were determined. Sleep health: hours of sleep and reparative sleep (RS) were examined using the question "How well do you usually sleep?" measured on a Likert scale (1=good [RS], 2=regular, 3=badly, $4=$ with medication/treatment (non-reparative sleep [NRS]). Function and pain were measured using the WOMAC and SF-36 questionnaires. Comparisons were made using t-tests (paired samples) and McNemar's test. Linear regression models were used to analyze associations. Dependent variables: WOMAC and SF-36 pain and function dimensions; independent variables: sleep quality, age, sex, BMI, number of comorbidities, depression/anxiety.

Results: 105 patients ( $79 \%$ female, mean age 69.39 years [SD 8.3]) were included. $80 \%$ had $\geq 2$ comorbidities (mean 2.71 [SD 1.8]), mean BMI was 33.68 (SD 6.7), 32 had depression/anxiety, and mean sleep duration was 6.63 hours (SD 1.4). 12 months after TKF there were significant improvements in WOMAC dimension scores (mean $>25$ points, $p<0.001$ ) and SF-36 scores (mean $>19$ ). At study inclusion, $23 \%$ reported RS with a mean sleep duration of 7.5 hours (SD 1.1 ) vs. 6.24 hours (SD 1.5) in NRS patients ( $p=0.002) .12$ months after TKR, $40 \%$ of patients had RS $(p=0.029)$. Patients with RS had better scores in all quality of life dimensions $(<10$ points) than those with NRS $(p<0.05)$ at baseline and at 12 months. Multivariate analysis showed RS was independently associated with pain and function (WOMAC and SF-36) $(p<0.007)$.

Conclusions: Sleep health was associated with better HRQL before and after TKR. Although more patients had RS after TKR, $60 \%$ of patients continued not to have sleep health. Although often undervalued clinically, sleep health is closely associated with the health status.

Acknowledgements: This work was funded by project $\mathrm{Pl} / 13 / 00948$, integrated in the Plan Nacional I+D+I and cofounded by ISCIII-Subdirección General de Evaluación and European Regional Development Fund (ERDF).

References:

[1] Buysse DJ. Sleep health: can we define it? Does it matter? Sleep. 2014 Jan 1;37(1):9-17. doi: 10.5665/sleep.3298.

Disclosure of Interest: None declared

DOI: 10.1136/annrheumdis-2017-eular.1777

\section{AB1151 MONTH OF BIRTH AFFECTS THE RISK OF RHEUMATIC DISEASES: A NATIONWIDE CASE-CONTROL STUDY}

N. Koo, J. Lee, B.W. Lee, S.-K. Kwok, S.H. Park, J.H. Ju. Division of Rheumatology, Department of Internal Medicine, School of Medicine, The Catholic University of Korea, Seoul St. Mary's Hospital, Seoul, Korea, Republic Of

Background: There have been several studies which demonstrated the impact of birth on the risk of certain diseases such as asthma or cardiovascular diseases.
However, rheumatic diseases have not yet been thoroughly investigated in terms of association with birth month.

Objectives: In this study, we sought to determine whether birth month or season could affect the risk of rheumatologic diseases.

Methods: The birth month patterns of patients with rheumatic diseases were compared with to those of general population. We utilized the claims data of Health Insurance Review and Assessment Service (HIRA) which covers nearly $90 \%$ of total population in Korea. The associations between birth month/season and 32 diseases were investigated using logistic regression.

Results: Our dataset included 17,247,458 (male 8,224,670; female 9,022,788) individuals from HIRA database from January, 1997 to August, 2015. Among 27 rheumatic diseases, 8 diseases including Crohn's disease (CD), ulcerative colitis (UC), rheumatoid arthritis (RA), systemic lupus erythematosus, polymyalgia rheumatica (PMR), ankylosing spondylitis (AS), multiple sclerosis, gout, fibromyalgia (FMS) were significantly associated with birth month $(P<0.05)$. In terms of seasonality, CD, UC, RA, Sjogren's syndrome, PMR, AS, Gout, and FMS demonstrated significant difference. CD, UC and AS showed higher prevalence in individuals born in winter and lower prevalence in summer. On the other hand, people who were born in summer showed higher possibility to have gout and FMS compared to those born in winter. In consistent with previous reports, type 1 diabetes is more prevalent in those born in winter. Angina and myocardial infarction showed higher prevalence in patients born in spring and lower in fall. This consistency reflects the relevance of our dataset and methodology.

Conclusions: We found significant impacts of birth month/season on various rheumatic diseases. Seasonal variation of infective agents, sun exposure or food ingestion during gestation or early infancy may explain the association between birth month/season and certain disease development.

Disclosure of Interest: None declared

DOI: 10.1136/annrheumdis-2017-eular.2891

\section{AB1152 THE INCIDENCE OF HYPERSENSITIVITY TO NSAIDS IN THE GROUP OF PATIENTS WITH MUSCULOSKELETAL DISORDERS}

O. Brzezińska-Pawłowska ${ }^{1}$, A. Sztanka ${ }^{2}$, K. Słodkowski $^{2}$,

A. Lewandowska-Polak ${ }^{2}$, J. Makowska ${ }^{2}$. ${ }^{1}$ Department of Rheumatology, Immunology and Allergy; ${ }^{2}$ Department of Rheumatology, Medical Univercity of Lodz, Łódź, Poland

Background: Non-steroidal anti-inflammatory drugs (NSAIDs) are among the most common cause of drug hypersensitivity reactions (DHR). Recent studies show that the prevalence of hypersensitivity reaction to drug is particularly in Poland.

Objectives: To assess the frequency and the risk od developing NSAID hypersensitivity in patients with chronic disorders of the musculoskeletal system. Methods: The study group consisted of 200 patients (age 19-88 years - $54,5 \pm 14$, women-161, men-39) hospitalized in the Department of Rheumatology in 20152016. All patients filled questionnaire regarding symptoms of DHR after ingestion of non-steroidal anti-inflammatory drugs. The presence of DHR, clinical pattern of the reaction, frequency of NSAIDs administration and comorbidities have been studied. In statistical studies the main risk factors were defined.

Results: Seventy-seven patients from study group (38,5\%) reported symptoms that occurred within 24 hours after NSAIDs ingestion. Symptoms characteristic for hypersensitivity reaction were reported by 40 patients $(20 \%)$. Respiratory symptoms like dyspnea and/or cough were reported by 22 patients $(11 \%)$. Cutaneous symptoms (urticaria/angioedema/dermal flush) were reported also by 29 patients $(14,5 \%)$. Three patients experienced loss of consciousness. Thirty-seven patients reported isolated stomach cramps.

The symptoms developed usually between 2-12 hours after drug intake. In 37 patients oral administration caused DHR reaction, moreover in 14 patients also topical application led to adverse reaction. In most of patients reaction appeared due to COX-1 inhibitor and interestingly in 3 patients reaction was evoked by celecoxib which is perceived to be safe alternative for patients with NSAIDs hypersensitivity. Chronic urticaria, asthma and systematic drug intake occurs the main risk factors of hypersensitivity.

Conclusions: Drug hypersensitivity reactions are reported very frequently in population of patients with chronic musculoskeletal disorders. Our studies suggest that patients taking protractedly NSAIDs are in group with high risk of drug hypersensitivity development.

Disclosure of Interest: None declared

DOI: 10.1136/annrheumdis-2017-eular.5408

\section{AB1153 OSTEOPOROSIS RISK FACTORS IN PARTICIPANTS OF HEALTHY AGING ACADEMY}

O. Brzezińska-Pawłowska ${ }^{1}$, A. Łuczak ${ }^{2}$, D. Stalmach ${ }^{2}$, A. Lentas ${ }^{2}$,

M.L. Kowalski ${ }^{1}$, J. Makowska ${ }^{2} .{ }^{1}$ Department of Immunology, Rheumatology and Allergy; ${ }^{2}$ Department of Rheumatology, Medical Univercity of Lodz, Łódź, Poland

Background: Osteoporosis is a major cause of morbidity and mortality in elderly population. It is known that the mortality rate in European population within 1 year of hip fracture is about $30 \%$, and another $30 \%$ of patients need long term nursing for the rest of their life. However, the prevalence and associated risk factors in the Polish elderly population have not been well documented. The aim of the 